TUMOUR METABOLISM

\title{
Suppressed fate
}

Pancreatic ductal adenocarcinoma (PDAC) is often characterized by co-occurring activating KRAS mutations and loss-of-function TP53 mutations. While mutant KRAS drives the appearance of premalignant precursor lesions, such as pancreatic intraepithelial neoplasia (PanIN), the presence of inactivating TP53 mutations is associated with progression to dedifferentiated malignant disease. Oncogenic KRAS is known to regulate metabolic pathways during pancreatic cancer growth yet the metabolic consequences of TP53 mutations are less well established. Therefore, to explore how p53-dependent metabolic changes might restrain tumour progression, Morris IV et al. adapted a mouse model of human PDAC.

As with traditional mouse models of PDAC, this one was generated by expressing $K R A S^{\mathrm{G} 12 \mathrm{D}}$ and inducing loss of p53 function specifically within the pancreas. However, to suppress p53 expression, short hairpin RNA (shRNA) targeting Trp53 controlled by doxycycline treatment (named KP ${ }^{\text {sh }}$ mice) was used instead of conditional deletion or mutation. The advantage of this system is that following tumour formation, endogenous p53 can be re-expressed through withdrawal of doxycycline.

Subsequently, the authors generated $\mathrm{KP}^{\mathrm{sh}}$ cell lines from tumours that developed in the modified mouse model to investigate PDAC metabolism following restoration of $\mathrm{p} 53$. Typically, cultured PDAC cells arising from co-occurring genomic alterations in KRAS and p53 use glucose and glutamine to sustain their proliferation. However, p53 induction in $\mathrm{KP}^{\text {sh }}$ cell lines, although resulting in cell cycle arrest, did not result in dramatic changes in the consumption of these two substrates.

A closer examination of the mitochondrial tricarboxylic acid (TCA) cycle revealed that there was a difference in downstream usage of carbons derived from the two substrates. p53 could initiate a metabolic switch whereby glucose-derived carbons were incorporated into TCA cycle intermediates, such as citrate and a-ketoglutarate ( $\alpha \mathrm{KG}$, also known as 2-oxoglutarate), at a higher rate and at the expense of metabolites originating from glutamine oxidation, such as succinate. The outcome of this metabolic rewiring was an augmented ratio of $\alpha \mathrm{KG}$ to succinate, which was independent of changes in proliferation and reversible upon re-administration of doxycycline.

p53 canonically functions to transcriptionally regulate downstream target genes and in doing so can modify cell fate and limit tumour development. In accordance with this role, re-introduction of $\mathrm{p} 53$ into $\mathrm{KP}^{\text {sh }}$ cells led to increases in the transcription of several TCA cycle enzymes including pyruvate carboxylase and isocitrate dehydrogenase 1 (IDH1). As both of these enzymes can act to increase aKG levels it seems likely they might contribute to the mechanism by which p53 increases the $\mathrm{\alpha KG}$ : succinate ratio.

Additionally, induction of p53 increased chromatin accessibility and resulted in premalignant patterns of gene expression consistent with p53 regulating transcriptional programmes associated with the transition from precursor lesions to malignant PDAC. In agreement with these in vitro observations, orthotopic tumours derived from $\mathrm{KP}^{\text {sh }}$ cells implanted into mice displayed a more differentiated histopathology following doxycycline withdrawal. Importantly, addition of cell-permeable $\alpha \mathrm{KG}$ alone to KPsh cells was sufficient to recapitulate chromatin and gene expression profiles observed upon p53 activation.

An increase in the $\alpha \mathrm{KG}$ : succinate ratio can also be achieved by inhibiting oxoglutarate dehydrogenase (OGDH), which metabolizes $\alpha$ KG. Similar to $\mathrm{p} 53$ re-expression or treatment with aKG, doxycycline-inducible shRNA-mediated knockdown of Ogdh in p53-null PDAC (KPflox)

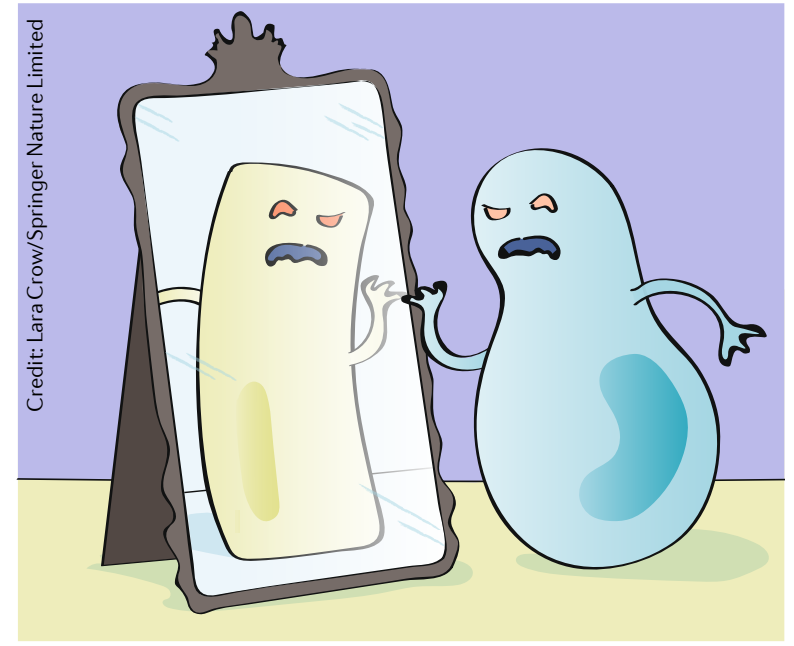

cells resulted in an accumulation of intracellular $\alpha \mathrm{KG}$ and enrichment of premalignant genes. When implanted into mice, these p53-null cells with suppressed OGDH expression formed smaller orthotopic tumours with a well-differentiated morphology compared with p53-null cells expressing control shRNAs.

Lastly, the authors assessed whether the activity of enzymes dependent on $\alpha \mathrm{KG}$ varied with respect to $\mathrm{p} 53$ status during PDAC development. They observed that levels of 5-hydroxymethylcytosine $(5 \mathrm{hmC})$, the chromatin modification arising from the activity of $\alpha \mathrm{KG}$ dependent TET enzymes, were reduced during progression from benign to malignant human PDAC, coinciding with loss of $\mathrm{p} 53$. Moreover, p53 restoration or addition of $\alpha \mathrm{KG}$ in $\mathrm{KP}^{\mathrm{sh}}$ cells or inhibition of OGDH in p53-null cells was capable of increasing $5 \mathrm{hmC}$ levels in vitro and in vivo. This indicates that $\alpha \mathrm{KG}$, through its accumulation, acts as a metabolic effector of p53-triggered tumour suppression coinciding with the induction of chromatin marks specific to premalignant differentiation.

As p53 has proved notoriously difficult to target therapeutically, this study suggests that strategies to indirectly target p53 through increasing the aKG: succinate ratio might serve to induce tumour cell differentiation and reverse cell identity to a premalignant state.

tumour cell differentiation Anna Dart

ORIGINAL ARTICLE Morris, J. P. IV et al. a-Ketoglutarate links $p 53$ to cell fate during tumour suppression. Nature 573, 595-599 (2019) 